\title{
Transcatheter Arterial Chemoembolization with Drug-Eluting Beads for the Treatment of Hepatocellular Carcinoma: Recommended Selection for Small-Caliber $(<100 \mu \mathrm{m})$ Beads
}

\author{
Wei-Chou Chang (ID) \\ Hsian-He Hsu' \\ Sung-Hua Chiu (iD) \\ Wen-Yen Huang ${ }^{2}$ \\ Cheng-Hsiang Lo ${ }^{2}$ \\ Hsuan-Hwai Lin ${ }^{3}$ \\ Pei-Ching Huang ${ }^{4}$ \\ Yu-Lueng Shih ${ }^{3}$ \\ Yung-Liang Wan iD ${ }^{4}$ \\ 'Department of Radiology, Tri-Service \\ General Hospital and National Defense \\ Medical Center, Taipei, Taiwan; \\ ${ }^{2}$ Department of Radiation Oncology, Tri- \\ Service General Hospital and National \\ Defense Medical Center, Taipei, Taiwan; \\ ${ }^{3}$ Division of Gastroenterology, \\ Department of Internal Medicine, Tri- \\ Service General Hospital and National \\ Defense Medical Center, Taipei, Taiwan; \\ ${ }^{4}$ Department of Medical Imaging and \\ Intervention, Linkou Chang Gung \\ Memorial Hospital, Chang Gung \\ University, Taoyuan City, Taiwan
}

\begin{abstract}
Drug-eluting beads transarterial chemoembolization (DEB-TACE) is an alternative to conventional lipiodol-based TACE (cTACE) to treat hepatocellular carcinoma (HCC). With the advancement in pharmacology, small-caliber DEB-TACE $(<100 \mu \mathrm{m})$ has been introduced since 2016. For the treatment of hepatic neoplasms or HCC, there is a tendency to use smaller beads by DEB-TACE to achieve more extensive tumor necrosis and a significant reduction in liver toxicity in comparison with that caused by cTACE. However, the indications and potential complications of small-caliber DEB-TACE remain uncertain and have not been well established, due to lack of randomized phase III clinical trials. Instead of systematic or meta-analysis review, this narrative review article describes the suggested indications and contraindications of DEB-TACE with small DEBs, benefit of super-selective embolization of the feeding arteries and the recommended selection of smallcaliber DEB. This review was approved by the institutional review board (File Number: 1-105-05-158)
\end{abstract}

Keywords: chemoembolization, HCC, TACE, DEB-TACE, liver, review

\section{Plain Language Summary}

Conventional lipiodol-based TACE (cTACE) has been used to treat hepatocellular carcinoma (HCC). However, due to advancement in pharmacology, drug-eluting beads transarterial chemoembolization (DEB-TACE) using small-caliber beads $(<100 \mu \mathrm{m})$ has been introduced to achieve more extensive tumor necrosis and a significant reduction in liver toxicity in comparison with that caused by cTACE.

\section{Introduction}

Transarterial chemoembolization (TACE) is primarily indicated in patients with hepatocellular carcinoma (HCC), chiefly in those with intermediate-stage disease but also in the early and, less frequently, advanced stages, based on the Barcelona Clinic Liver Cancer (BCLC) guidelines. Although conventional TACE (cTACE) with selective injection of lipiodol mixed with chemotherapeutic agents (doxorubicin or cisplatin) followed by vascular embolization constitutes the largest portion of transarterial treatment for HCC. The introduction of drug-eluting beads (DEBs) in the early 2000s provided an alternative to lipiodol-based regimens. Moreover, chemotherapeutic agents may be varied in different countries and institutions, cTACE has major
Correspondence: Yung-Liang Wan Department of Medical Imaging and Intervention, Linkou Chang Gung Memorial Hospital, Chang Gung University, Taoyuan City, Taiwan Emailylw0518@cgmh.org.tw 
drawbacks due to inter-operator variance and the lack of a universal drug delivery approach. In recent years, there has been increasing clinical evidence that DEBs have a reduced cytotoxic drug-related toxicity and liver toxicity in a sustained manner as compared with cTACE $^{1}$ with low systemic toxicity. With the advancements in drug development, different sizes of DEBs have been introduced into interventional radiology, and the size of commercially available DEBs varies considerably, ranging from 40 to $700 \mu \mathrm{m}$.

In the era of using DEBs larger than $100 \mu \mathrm{m}$, the patients had higher overall survival and lower toxicity of TACE using small (100-300 $\mu \mathrm{m})$ DEBs in comparison with those using large (300-500 and 500-700 $\mu \mathrm{m}$ ) DEBs, particularly in patients with advanced HCCs with relatively poor liver function (Child-Pugh classes $B$ and C). ${ }^{2,3}$ Patients treated with smaller $(<100 \mu \mathrm{m})$ DEBs are significantly associated with improvement with respect to the rates of treatment response, procedure-related complications and post-embolization syndrome. ${ }^{4-6}$ However, in comparison with cTACE, the use of such smaller $(<100$ $\mu \mathrm{m})$ DEBs is associated with increased risk of hepatobiliary injuries, such as bile duct dilatation, portal vein narrowing, and hepatic failure. ${ }^{4-6}$

The use of small-caliber beads $(<100 \mu \mathrm{m})$ enables more distal embolization, greater penetration, and a higher drug concentration in the network of neoplastic vessels. $^{7,8}$ In this narrative review, we summarize the updates on DEB-TACE and present the potential benefits of using small-caliber DEB (which is defined as beads smaller than $100 \mu \mathrm{m}$ in this article) to treat HCC.

\section{Mechanism of DEB-TACE and Its Pharmacokinetics}

To date, at least four types of commercially available DEB have been developed (Table 1). ${ }^{9,10}$ These are all negatively charged and can be loaded with different chemotherapeutic agents. Doxorubicin is the most popular loaded chemotherapeutic drug used for DEB-TACE treatment of HCC. Although treatment recommendation remains controversial among the interventional radiologists, it can be basically recommended for HCC patients within or beyond the Milan criteria: ${ }^{11,12}$

1. Patients within the Milan criteria: a planned dose of 50 to $75 \mathrm{mg}$ of doxorubicin is loaded into one vial for each single treatment.
2. Patients beyond the Milan criteria: a planned dose of up to $150 \mathrm{mg}$ of doxorubicin is loaded into two vials for each single treatment. For a huge tumor with large diameter $(>8 \mathrm{~cm})$ or bilobar tumors, treatment typically includes separate sessions (in intervals of 4 to 8 weeks, in the presence of complications, a longer time interval is required).

Uptake of doxorubicin onto the beads occurs between 30 minutes and 4 hours (small beads load more quickly due to surface area effects). The time required to load $99 \%$ of doxorubicin is defined as the loading time. The loading time varies for different beads, and for a given bead, different sizes can also cause different loading times. ${ }^{13}$ The smaller the bead size, the faster is the drug loading time. ${ }^{14}$ After loading, DEBs will be infused into tumors from the catheter or microcatheter, and doxorubicin will elute from the beads. The eluting time is the time taken to elute $75 \%$ of the total released doxorubicin. Then, doxorubicin will penetrate into tumors, and a small percentage of them will inevitably go into the systemic circulation. It is known that the plasma concentration of doxorubicin in TACE is related to the systemic toxicity. The use of DEBTACE has been associated with a significantly lower plasma concentration of doxorubicin as compared with cTACE.$^{15}$ Moreover, DEB-TACE can be used to continuously release doxorubicin, even at 2-3 weeks after embolization. ${ }^{16}$

\section{Indications and Contraindications for DEB-TACE with Different Bead Sizes}

In general, indications and contraindications for using DEB-TACE are almost the same as those for TACE. ${ }^{9}$ The PRECISION V study ${ }^{17}$ proposed that DEB-TACE is superior to cTACE for patients with advanced disease, poor heart function, and impaired liver function. However, with regard to TACE-related hepatic locoregional complications, DEB-TACE has been associated with a higher risk for complications than cTACE, and the latter may be a better option for patients with high baseline prothrombin value. $^{18}$

Within the DEB-TACE, different sizes of beads have variable levels of therapeutic efficacy and safety profiles. Currently, there is no consensus on the optimal particle size for DEB-TACE. Three types of drug-eluting beads are $100-300 \mu \mathrm{m}$ in size after loading with doxorubicin: (1) 


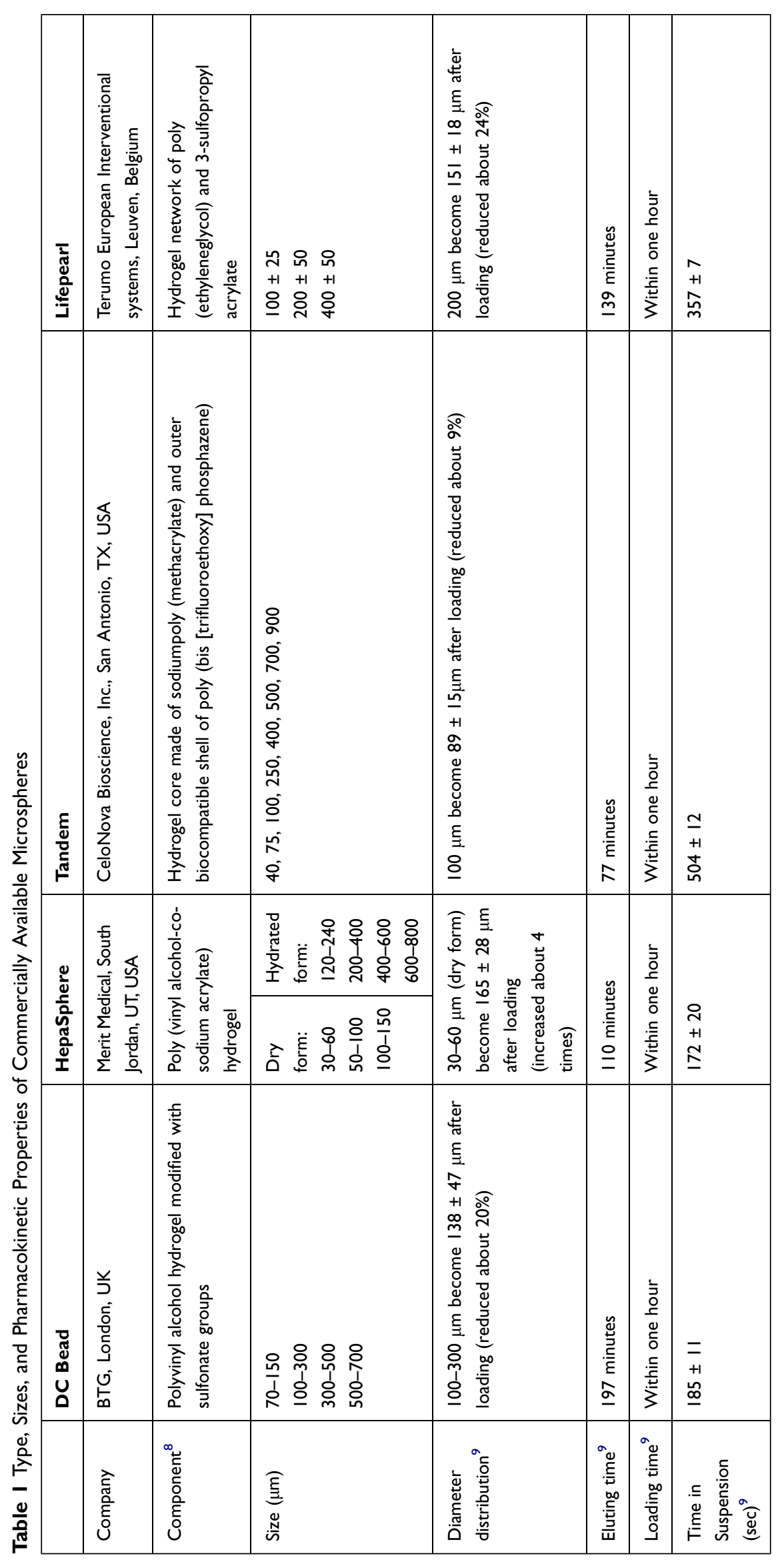


100-300 $\mu \mathrm{m}$ DC beads, (2) 30-60 $\mu \mathrm{m}$ Hepasphere, and (3) the $200 \mu \mathrm{m}$ Lifepearl. It is known that $100-300 \mu \mathrm{m}$ DC beads have a better therapeutic effect and lower complications than larger beads. ${ }^{2}$ In animal models, 30-60 $\mu \mathrm{m}$ Hepasphere showed a denser distribution of microspheres, a higher tissue and equal plasma concentration of doxorubicin in comparison with the 120-240 $\mu \mathrm{m}$ Hepasphere. ${ }^{19}$ When administrated to HCC patients, TACE with 30-60 $\mu \mathrm{m}$ Hepasphere beads had an acceptable level of therapeutic efficacy with a complete response rate of $17.8 \%$, and no 30-day mortality was encountered. ${ }^{20}$ For the Lifepearl, limited studies have focused on its therapeutic efficacy and safety. The PARIS registry, ${ }^{21}$ which studied both 100 $\mu \mathrm{m}$ and $200 \mu \mathrm{m}$ Lifepearl beads, showed a higher tumor response rate and lower hepatobiliary toxicity with these beads in comparison with those from cTACE.

A limited number of studies have focused on smallercaliber DEBs. The use of the DEB-TACE with $70-150 \mu \mathrm{m}$ DC beads and 100-300 $\mu \mathrm{m}$ DC beads was associated with greater hepatobiliary toxicity than when $100-300 \mu \mathrm{m} \mathrm{DC}$ beads were used alone, with no difference in tumor response. ${ }^{22}$ A retrospective study with 51 patients showed a better one-month objective response rate for $70-150 \mu \mathrm{m}$ DC beads in comparison with $100-300 \mu \mathrm{m}$ DC beads, without differences in overall survival or the safety profile. $^{23}$ A retrospective study with 72 HCC patients treated with idarubicin-loaded Tandem beads (75 and 100 $\mu \mathrm{m})$ reported an objective response rate of $65 \%$, a median time-to-treatment failure of 14.4 months, and a median overall survival time of 34.6 months. ${ }^{24}$

The primary concern regarding chemoembolization with small-caliber DEBs in HCC is safety, especially in terms of pulmonary embolization, tumor rupture, and biliary damage, despite the fact that it is more effective than TACE treatments with larger beads.

\section{Modified Response Evaluation Criteria in Solid Tumors (mRECIST) to Evaluate Treatment Response and Reasons for Tumor Viability Escape from TACE}

The clinically mRECIST criteria are widely used for the assessment of tumor response after HCC treatment ${ }^{25}$ and have been proven to have a strong prognostic correlation in HCC patients undergoing logo-regional treatment. ${ }^{26}$ In mRECIST, the treatment response depends on intratumoral arterial enhancement rather the whole-tumor size measured in RECIST. It has been proven that the presence of a complete response after the initial TACE treatment is a good prognostic predictor, ${ }^{27}$ because post-TACE viable tumors are prone to becoming TACE-resistant, which is probably caused by the following three mechanisms:

\section{Sarcomatous Changes in the Viable Tumor After TACE}

Sarcomatous differentiation is a poor prognostic factor for HCC patients. ${ }^{28}$ It was found in $4.2 \%$ of treatment-naïve HCC patients and $20.9 \%$ of HCC patients undergoing anticancer therapy. ${ }^{29}$ Of these cases, sarcomatous change was most frequently observed in patients with repeated transarterial embolization.

\section{Presence of the Hepatocholangiocellular Phenotype}

In a study involving $80 \mathrm{HCC}$ nodules, ${ }^{30}$ including 40 treated with TACE and 40 not treated with locoregional therapy (LRT) removed during liver transplantation, 35\% of the TACE-treated nodules showed the hepatocholangiocellular phenotype, and none of the nodules had this phenotype after LRT.

\section{Tumor Blood Supply from the Portal Vein} A study involving 12 HCCs treated with TACE showed that the portal blood supply to TACE-resistant tumors became apparent on computed tomography (CT) images in the portal venous phase, ${ }^{31}$ which may have been caused by arterial damage by TACE. In short, avoiding residual viable tumors in the first round of TACE treatment is critical for the treatment of unresectable HCC.

Despite promising value of mRECIST is assessing treatment response of TACE, there are some limitations with inevitable major bias. ${ }^{32,33}$ For example, the interpretation of mRECIST relies on the observer consistency (inter- and intra-observer reproducibility). It is essentially not appropriate to use mRECIST to assess the sequential changes of arterial enhancement and diameters of the target HCC with extrahepatic metastases or HCC manifesting poor enhancement, infiltration, or high-grade portal vein invasion. ${ }^{25}$ Clinically, many patients are not able to have a series of follow-up imaging studies for assessment by mRECIST, and this could lead to a bias according to the World Health Organization criteria in deciding treatment discontinuation. ${ }^{32}$ 


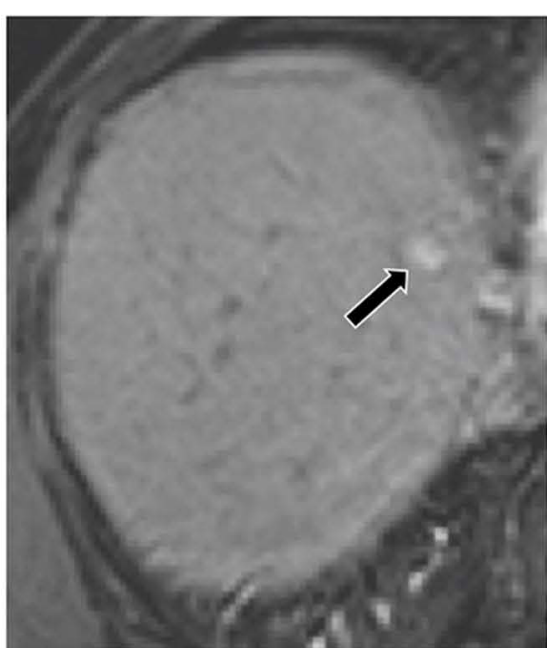

A

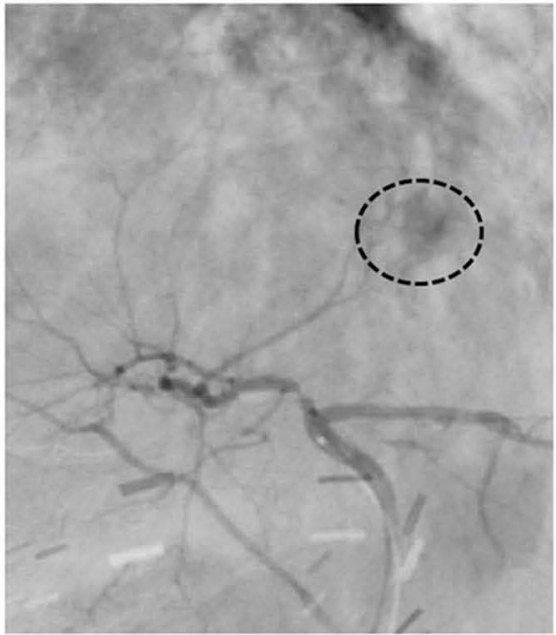

B

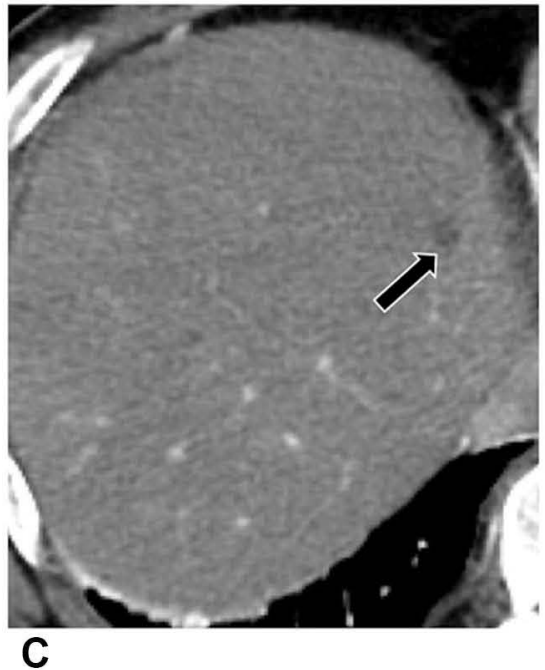

Figure I A small HCC supplied by small hepatic arteriole in a 62-year-old male. (A) Contrast-enhanced arterial-phase MR image shows a recurrent $\mathrm{HCC}$ in the segment 4 (black arrow). (B) Left hepatic angiogram demonstrates a faint hypervascular tumor in the segment 4 of liver (dotted circle) supplied by the superior branch of left hepatic artery. DEB-TACE was then performed. (C) Follow-up CT one month after TACE reveals no residual enhancement (black arrow). The patient was disease free without evidence of tumor recurrence to date.

\section{Superselective Embolization of the Tumor Feeding Arteries}

By definition, superselective TACE indicates management at the distal portion of the tumor-feeding subsegmental artery, in which microcatheter is engaged more distally than in selective TACE (management at the hepatic arterial branch afferent to the segment in which the tumor is located). Selective/superselective TACE has shown its therapeutic superiority compared with lobar TACE (management at the right/left hepatic artery) ${ }^{34}$ in cTACE, in which $53.8 \%$ of selective/superselective TACE-treated HCC nodules reached compete necrosis in comparison with $29.8 \%$ treated with lobar TACE. In advance, ultraselective TACE, in which a microcatheter is inserted into the tumor feeding artery, leaving a peritumoral safety margin in the liver parenchyma of at least $1 \mathrm{~cm}$, has been applied with cTACE for the treatment of HCC tumors smaller than $5 \mathrm{~cm}^{35}$ For DEB-TACE, superselective TACE treatments can assure that beads will be infused into tumors without compromising normal liver parenchyma to a great extent.

\section{Recommended Selection for Small- Caliber $(<100 \mu \mathrm{m})$ DEBs}

The optimal bead size for HCC patients receiving DEBTACE remains uncertain and has not been well investigated. There is no well-established indication for the selection of small-caliber DEBs in TACE treatment of HCC due to lack of multi-center Phase II or III clinical trials. The use of commercially available small-caliber DEBs mainly depends on the experiences of interventional radiologists.

The most commonly used beads in clinical practice are the DC Bead and HepaSphere. There is a tendency to use smaller beads to achieve more extensive tumor necrosis. In the era before the launch of small-caliber DEBs, a study ${ }^{3}$ reported that 100-300 $\mu \mathrm{m}$ doxorubicin DEBs are superior to $300-500 \mu \mathrm{m}$ for HCC treatment in terms of achieving a more complete imaging response at initial follow-up. Once again, a more recent study ${ }^{23}$ demonstrated that 75 $150 \mu \mathrm{m}$ doxorubicin DEBs are superior to $100-300 \mu \mathrm{m}$ ones in terms of achieving a tumor response. While differences in DEB-TACE techniques across centers still exist, based on our knowledge and experience, the recommended selection for small-caliber DEBs is as follows:

\section{Small HCCs Supplied by Small Hepatic Arteriole}

For small HCCs, 100-300 $\mu \mathrm{m}$ DC beads have shown their therapeutic efficacy as a bridge to liver transplantation. ${ }^{36} \mathrm{In}$ theory, the smaller size beads are characterized by a greater surface area and volume that could be delivered to the tumor before stasis, which consequently induces irreversible ischemia in the liver and biliary system. Super-selective DEBTACE with small-caliber beads (Figure 1) may spare a larger area of peritumoral hepatic parenchyma and reduce the phenomenon of hypoxic-ischemic neo-angiogenesis, but such a treatment may increase the incidence of biliary tract injury. 


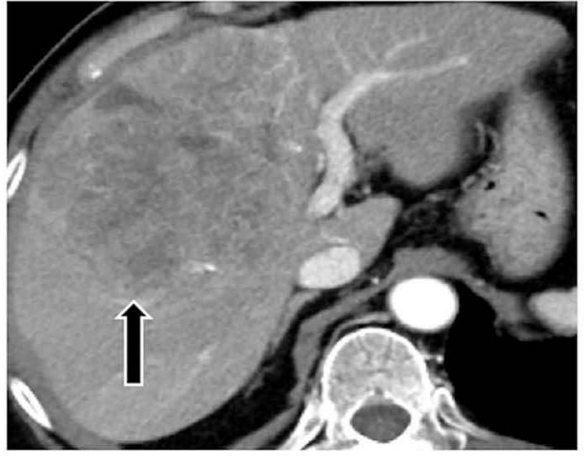

A

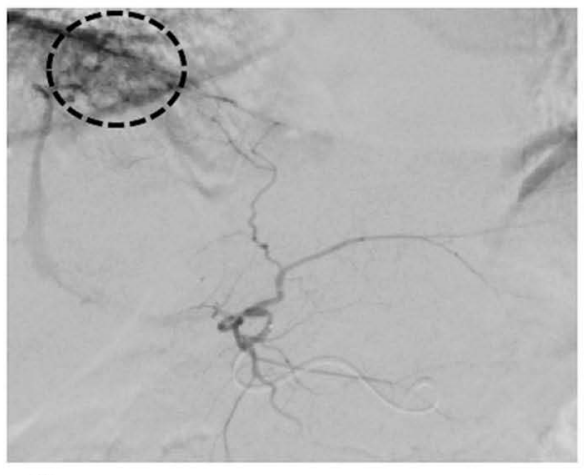

C

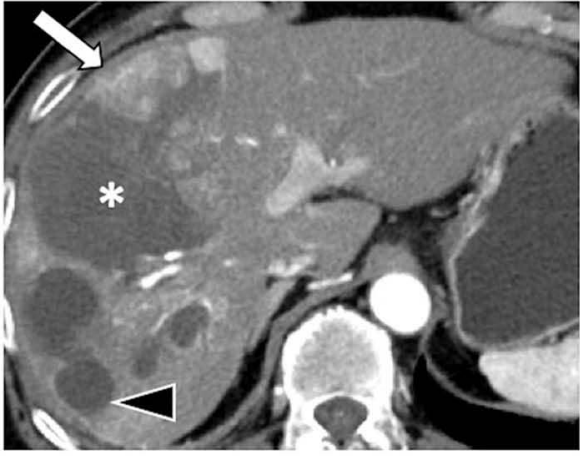

B

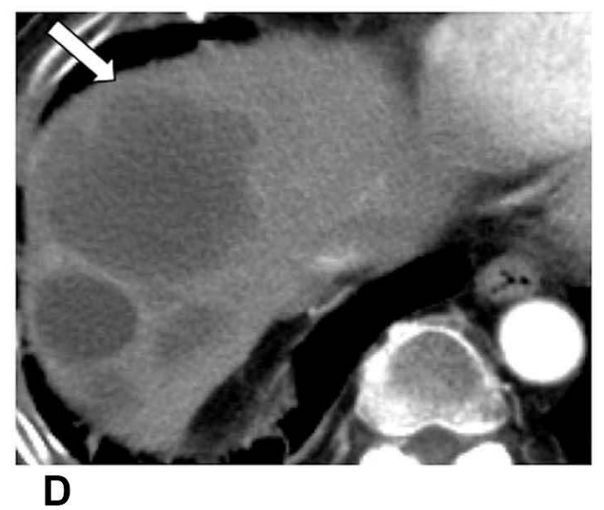

Figure 2 Repeated TACE for neovascularity of tumor-feeding arteries. An 80-year-old male with a large HCC in the segments 4/8. (A) A CT image in arterial phase CT shows a heterogeneous enhancing mass (black arrow). TACE with large-caliber DEBs was performed. (B) Follow-up CT 3 months after the Ist TACE reveals that approximately $80 \%$ of the HCC $(*)$ is necrotic with residual enhancement (white arrow), indicative of partial response. Biloma after the Ist TACE is depicted (black arrowhead). (C) Left hepatic angiogram during the 2nd TACE demonstrates residual tumors (dotted circle) supplied by newly-developed feeding arteries. TACE with smallcaliber DEBs was performed. (D) Follow-up CT 3 months after the 2nd TACE reveals necrosis of the entire tumor (white arrow) without residual enhancement.

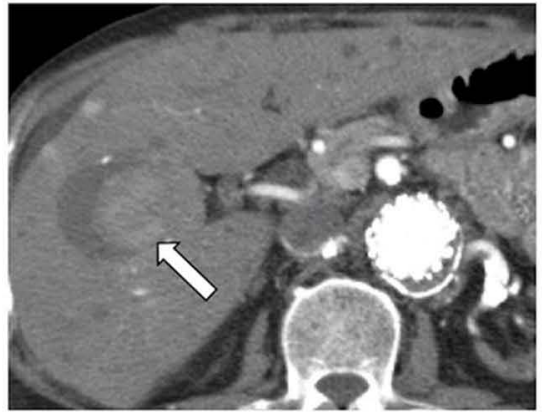

A

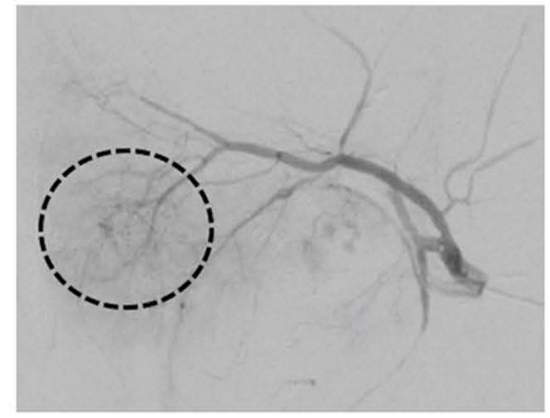

B

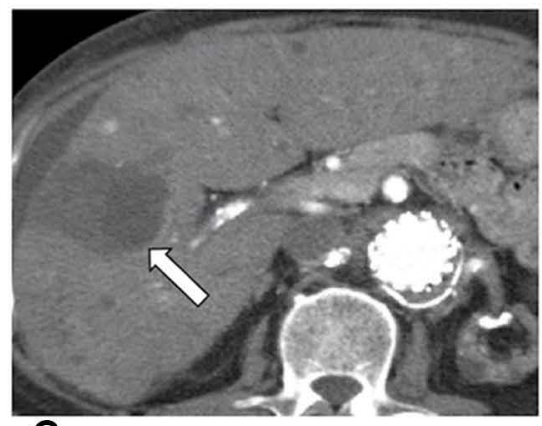

C

Figure 3 Another TACE treatment for residual viable tumor. A 67-year-old male with a previously treated HCC. (A) Follow up CT one year after the Ist TACE reveals partial necrosis of the HCC with residual enhancement (white arrow). (B) An angiogram during the 2nd TACE treatment reveals residual tumor with feeding arteries (dotted circle) originating from the right hepatic artery. TACE with small-caliber DEBs was then performed. (C) CT image three months after the 2nd DEB-TACE treatment shows no residual enhancement in the HCC (white arrow).

\section{Repeated TACE for Neovascularity of Tumor-Feeding Arteries}

Repeated TACE may deteriorate liver function ${ }^{37}$ and stimulate new tumor vessel formation, which is associated with tumor recurrence after TACE. The new tumor vessels produced due to angiogenesis induced via vascular endothelial growth factor are relatively small, very tortuous, and morphologically and functionally different from normal liver vessels. In repeated or multiple sessions of DEB-TACE, small-caliber DEBs are a better treatment option than large-caliber DEBs to treat neovascularity of tumor-feeding arteries (Figure 2). 


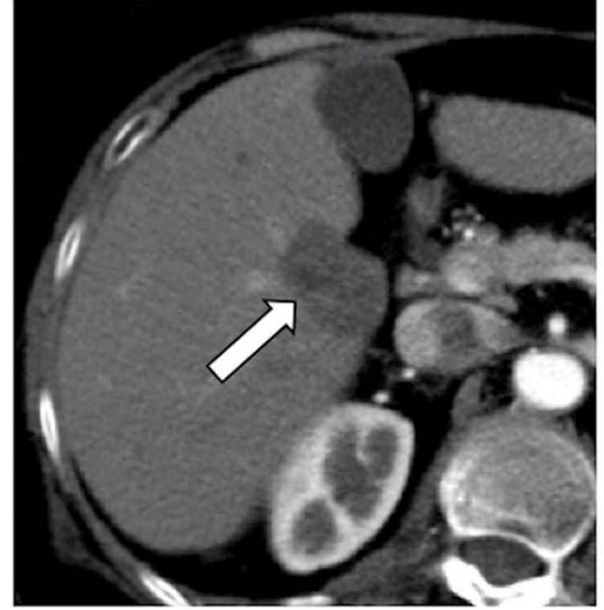

A

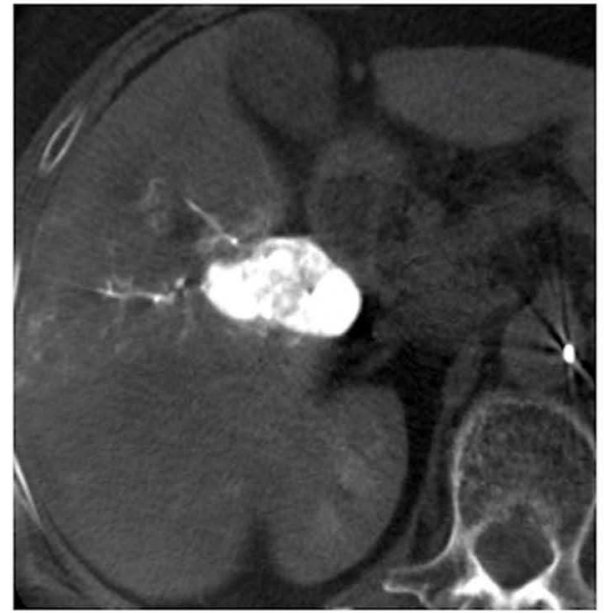

C

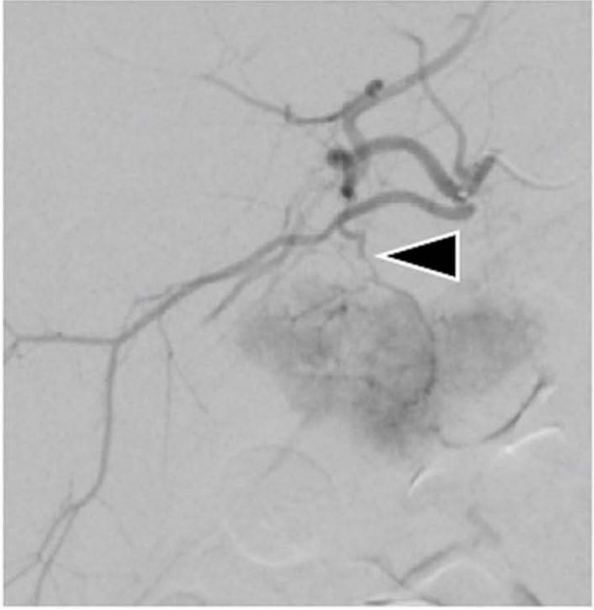

B

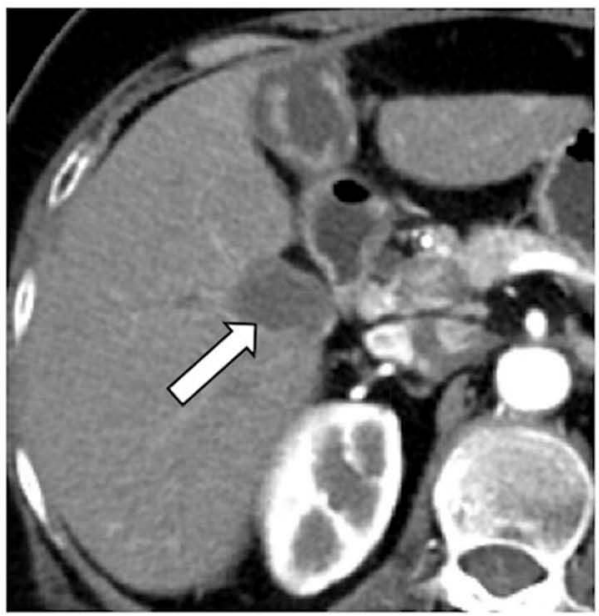

D

Figure $4 \mathrm{HCC}$ simultaneously treated with DEB-TACE and local radiofrequency ablation (RFA). A 7I-year-old male with a HCC near the hepatic hilum, which was treated with RFA. (A) Three months after RFA, CT shows a residual HCC (white arrow) in the right lobe of liver near hepatic hilum. (B) During TACE, the right hepatic angiogram reveals a hypervascular tumor supplied by a small branch of right hepatic artery (black arrowhead). (C) Post-TACE cone-beam CT image after small-caliber DEBs infusion shows homogeneously high attenuation of the residual HCC. (D) Follow-up CT one month after the TACE shows no evidence of residual enhancement (white arrow).

\section{Another TACE Treatment for Residual Viable Tumors}

It is unlikely for a single session of TACE to obtain a complete response for large HCC tumors. In order to achieve better local tumor control, switching small-caliber DEBs to another TACE treatment is recommended if the large HCC tumors are refractory to TACE (Figure 3).

\section{Simultaneous Use of DEB-TACE and Local Radiofrequency Ablation (RFA)}

The use of DEB-TACE combined with local ablative therapies has achieved excellent results for HCC treatment, with improved local tumor control and overall survival. RFA therapy has its limitations in terms of the size and number of HCCs it can treat, and some locations in the liver might be difficult to approach with ultrasonography guidance. TACE is likely to be used as a palliative treatment, as the local recurrence rate of small HCCs after DEB-TACE treatment has been reported to be up to $45.7 \% .{ }^{38}$ In order to produce full necrosis or to treat local recurrences of the target HCC, the combination of DEBTACE and RFA yields a better local tumor control and decreases tumor recurrence than each treatment alone (Figure 4).

\section{Extrahepatic Collateral (EHC) Blood Supply to HCC}

As exophytic growth of HCCs is usually located adjacently to the bare area of the liver and is treated by 




A

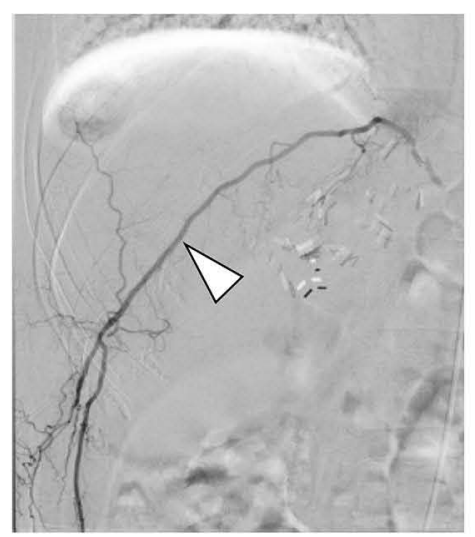

B

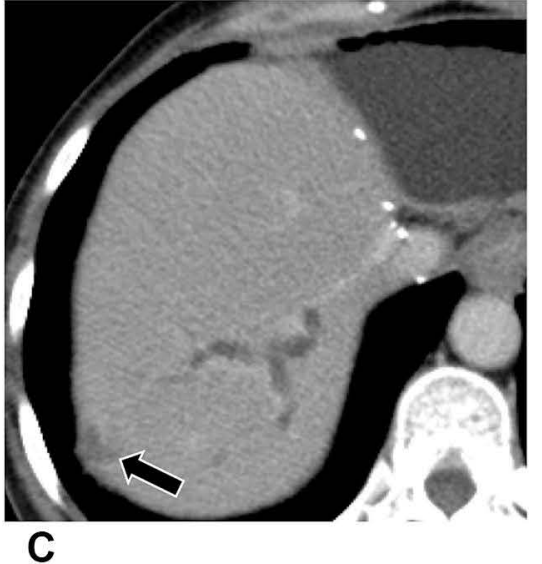

Figure 5 Extrahepatic collateral blood supply to HCC. A 46-year-old female with history of HBV-related HCC in the left lobe was treated with wedge resection. (A) Three years after the surgery, CT shows a recurrent HCC (black arrow) in the subcapsular region of segment 7 of liver. Focal dilatation of intrahepatic bile duct (black arrowhead) is depicted, which may be due to postoperative change. (B) During TACE, the right I2th lumbar angiogram (white arrowhead) reveals a hypervascular tumor in the right lobe of liver. Subsequently, TACE was performed with small-caliber DEBs. (C) Sixth month after DEB-TACE, the follow-up CT shows no residual enhancement of the entire HCC (black arrow).

LRT, $^{39}$ they may recruit the EHC blood supply. Most studies focusing on using TACE through EHC arteries have been limited to cTACE, ${ }^{40}$ and a retrospective study with 42 HCC patients treated with 30-60 $\mu \mathrm{m}$ Hepasphere beads through EHC arteries showed that DEB-TACE has a potential therapeutic effect on HCC tumors with an EHC blood supply, with a comparable safety level to cTACE. ${ }^{41}$ Small-caliber DEBs can be super-selectively delivered to more distal branches of these EHC arteries, preventing non-target embolization of non-liver organs (Figure 5).

\section{Patients with Poor Liver Function (Child- Pugh Score 8-9)}

DEB-TACE has been associated with a significant reduction in liver toxicity in comparison with that caused by cTACE. ${ }^{17}$ Moreover, DEB-TACE with small-caliber DEBs potentionally benefits HCC patients with poor liver function due to superselective embolization of a more focused liver area. Small-caliber DEBs can be delivered more distally by sparing non-tumoral cirrhotic liver parenchyma as much as possible to decrease liver toxicity (Figure 6).

\section{HCC with Relatively Low or Small Vascularity}

When an HCC features a low contrast medium enhancement on a dynamic CT or MRI in the arterial and portal venous phases, it is defined as HCC with relatively low or small vascularity (Figure 6). ${ }^{42}$ To date, few studies have focused on the TACE treatment of HCC with relatively low or small vascularity.

A single-center series enrolled 98 patients with atypical HCC (including relatively low or small vascularity and welldifferentiated $\mathrm{HCC}$ ) proposed decreased efficacy of TACE in these patients. ${ }^{43}$ Another single-institution retrospective study showed that DEB-TACE had better therapeutic efficacy and equivalent safety to cTACE. ${ }^{44}$ HCC with relatively low or small vascularity indicates less tumoral blood supply, and in this circumstance, small-caliber DEB can penetrate into tumor through small-sized tumor-feeding arteries, lead to better saturation of target tumors.

\section{Contraindications of Small-Caliber DEBs}

Portal vein thrombosis (PVT) with and without intrahepatic shunt are poor prognostic factors for HCC patients. Due to the high risk of developing hepatic failure, treatment with TACE is sometimes contraindicated. There is still uncertainty about the lower limitation of effective bead size, because the presence of PVT and intrahepatic shunts with smaller DEBs increases the risk of disease progression and complications (Figure 7).

\section{Follow-Up Imaging with mRECIST in HCC Patients Treated with cTACE and DEB-TACE}

Follow-up imaging is essential for evaluating the treatment response and determining whether further therapy is required. The recommendation of the mRECIST criteria, ${ }^{25,45,46}$ based 


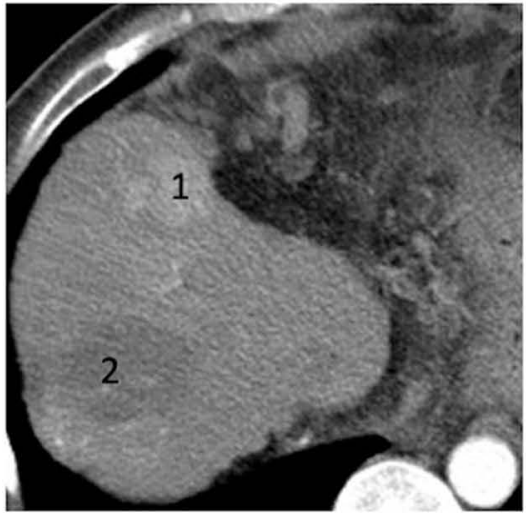

A

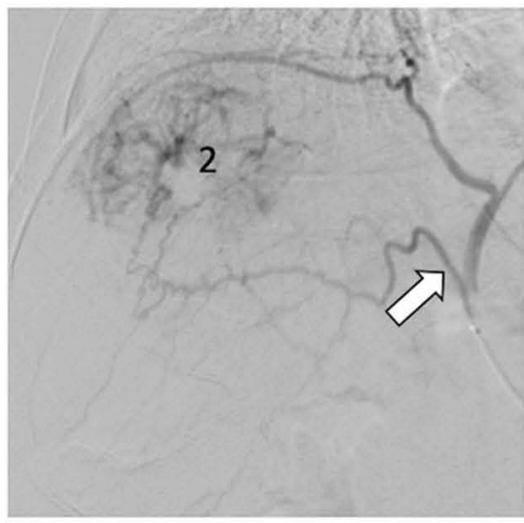

D

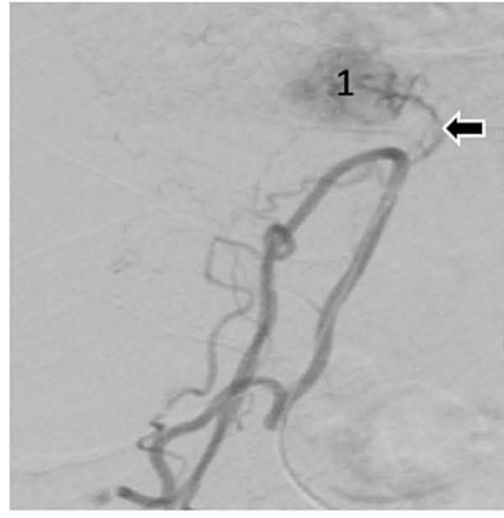

B

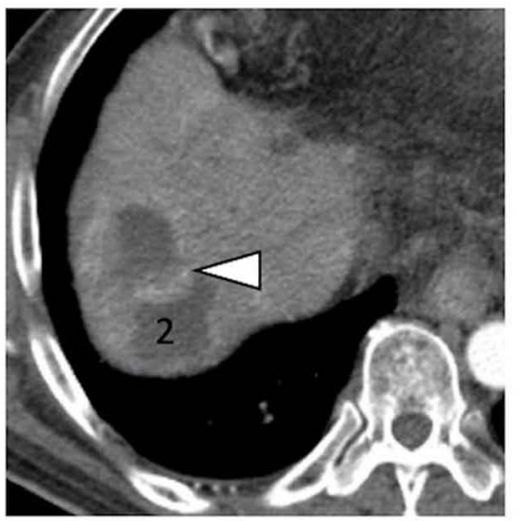

$\mathbf{E}$

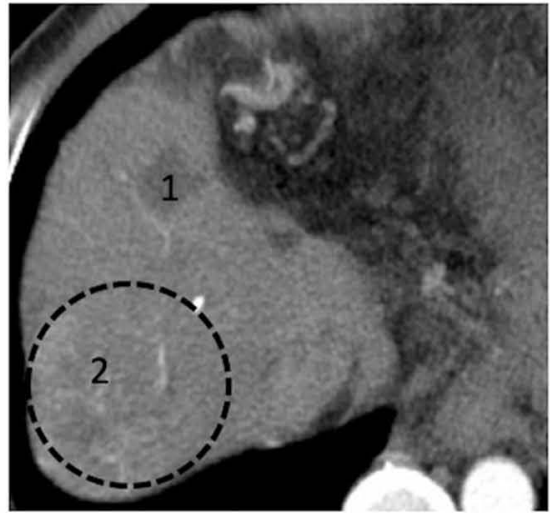

C

Figure 6 A 60-year-old HBV carrier had HCC in the left lobe which was treated with extended left lobectomy. He has poor liver function (Child-Pugh score 8-9) and HCC with relatively small or low vascularity. (A) CT image in arterial-phase shows two recurrent HCCs (tumor I and 2). The tumor I featured early arterial enhancement, and tumor 2 was not hypervascular suggestive of a HCC with relatively small or low vascularity. (B) An angiogram during the Ist TACE reveals a hypervascular tumor (tumor I) which is supplied by the right colic artery (black arrow, a branch of superior mesenteric artery). TACE with small-caliber DEBs of right colic artery was performed. (C) Three months after the Ist TACE, follow-up CT shows no evidence of residual enhancement of the tumor I lesion. However, the tumor 2 lesion features progression of tumor size with mild increase of the tumor vascularity (dotted circle). (D) An angiogram during the 2nd TACE shows that the tumor 2 is supplied by right inferior phrenic artery (white arrow) with low vascularity. TACE of right inferior phrenic artery was performed with small-caliber DEBs. (E) Three months after the 2 nd TACE, CT image shows marked cystic change of the tumor 2 with residual band-like enhancement (white arrowhead) suggesting partial response. The tumors were downgraded and the patient subsequently received liver transplantation.

on the one- or two-dimensional measurement of the enhanced portions of target tumors, has been advocated for the assessment of HCC after TACE treatment. In this criteria, if a target HCC that is solid at baseline becomes entirely necrotic and features no arterial enhancement after TACE, the treated HCC is classified as having complete response. Gillmore et $\mathrm{al}^{47}$ reported that the overall response measured based on the mRECIST within the first 3-month interval after TACE is independently related to survival.

The CT images of HCC of cTACE could be different from those after DEB-TACE (Table 2). After cTACE treatment, HCC tumors may feature a highly attenuated lipiodol deposition on unenhanced CT images, the distribution and pattern of retained lipiodol may be used to predict the therapeutic response. A triphasic contrast-enhanced CT is required to assess residual tumors that may be located near the retained lipiodol area. Retained lipiodol after cTACE may cause beam hardening artifacts and obscuration of residual tumors on CT images. Unlike cTACE, residual tumors on CT images in the arterial phase are more clearly delineated after DEB-TACE because of necrotic changes. Perilesional hyperenhancement after cTACE or DEB-TACE may be present and should not be misinterpreted as residual tumor, because this finding does not show portal venous washout and is typically circumferential rather than nodular.

MRI is superior to CT due to the absence of beam hardening artifacts and the better distinction of residual tumors. ${ }^{48}$ It also provides both anatomical and functional information about HCC tumors treated with TACE. The appearance of treated lesions can be variable in terms of signal intensity on T1-weighted and T2-weighted (T2W) images due to the presence of hemorrhage, cystic necrosis, and inflammation. ${ }^{49}$ 


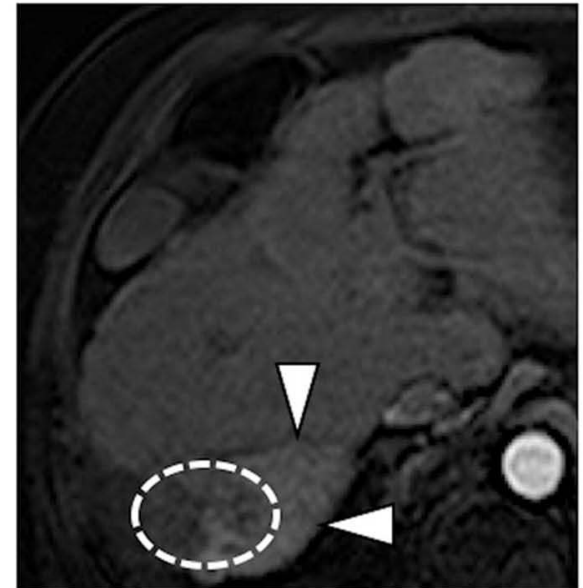

A

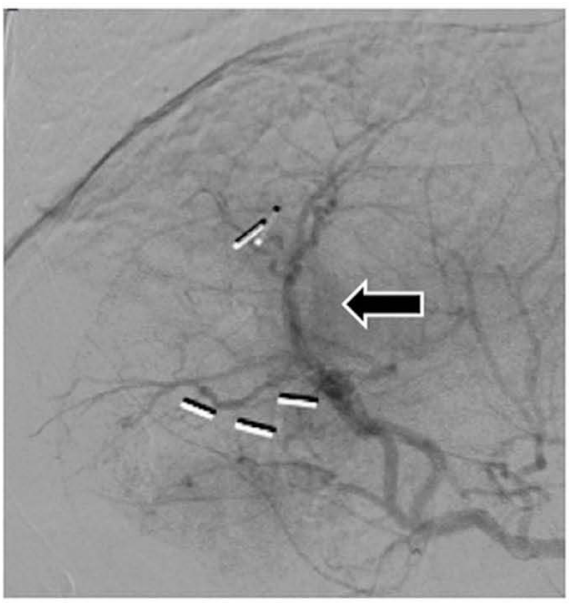

C

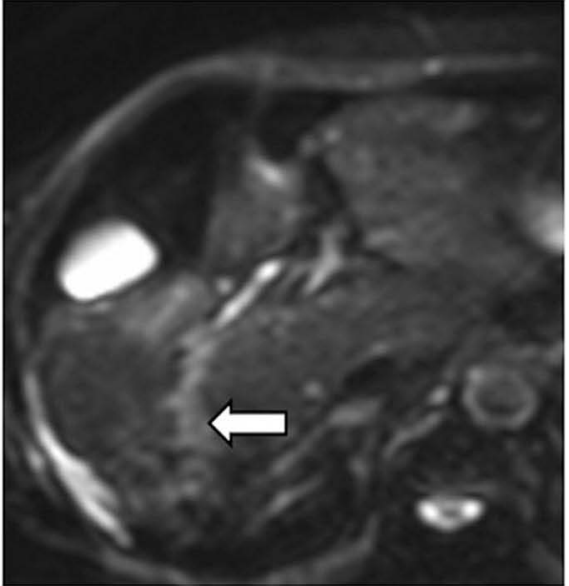

B

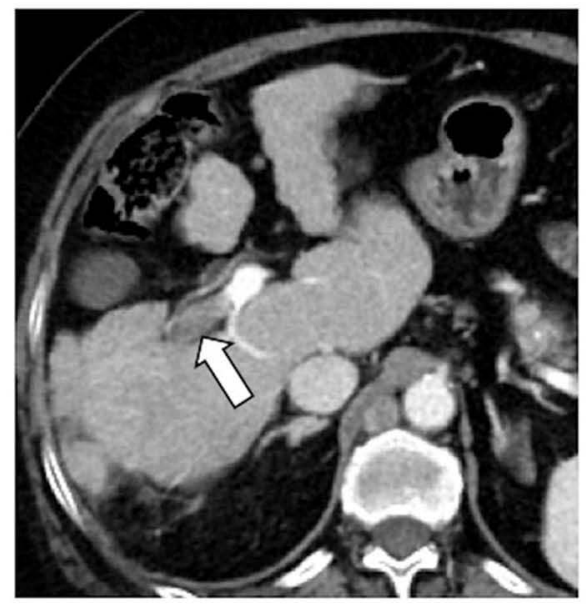

D

Figure 7 Contraindication of TACE with small-sized DEB: HCC with arterio-portal shunt and portal vein tumor thrombosis. A 69-year-old female with HCC in the segment 7 of liver was treated by stereotactic body radiation therapy. (A) Arterial-phase MR image shows a HCC (dotted line) with transient difference of the hepatic attenuation around the tumor (white arrowheads) probably due to arterio-portal shunt. (B) Diffusion-weighted MR image shows evidence of tumor thrombosis in the distal portion of right portal vein (white arrow). (C) During TACE, the right hepatic angiogram reveals early draining vein (black arrow) near the HCC. TACE with small-caliber DEBs was performed. (D) Follow-up CT 3 months after the TACE shows progression of the tumor thrombosis in the central portion of right portal vein (white arrowhead).

Residual tumors typically feature mild to moderate hyperintensity on $\mathrm{T} 2 \mathrm{~W}$ images. ${ }^{50}$ The necrotic cells in treated HCC tumors are associated with increased cell membrane permeability, mobility of water molecules, and ADC values. ${ }^{51}$ Both diffusion-weighted images and contrast-enhanced MRI may show early changes associated with the therapeutic response. ${ }^{52}$

\section{Conclusion and Future Perspectives}

DEB-TACE is effective and safe for the treatment of HCC, and interventions with small-caliber DEBs $(<100 \mu \mathrm{m})$ is recommended, especially in the following situations: 1) Small HCC supplied by small hepatic arterioles; 2) Repeated TACE for neovascular tumor-feeding arteries; 3) Repeated TACE for residual viable tumors; 4) Combined use of DEB-
TACE with local radiofrequency ablation; 5) Extrahepatic collateral blood supply to HCC tumors; 6) Patients with poor liver function (Child-Pugh score of 8-9); and 7) HCC with low or small vascularity. In comparison with DEB-TACE using larger-caliber beads, TACE treatment with smallcaliber DEBs $(<100 \mu \mathrm{m})$ may theoretically offer more complete embolization of the tumor with a better local tumor response and a lower recurrence rate. TACE treatment with small-caliber DEBs $(<100 \mu \mathrm{m})$ is indicated for super-selective embolization of feeding arteries in order to prevent major complications. It is necessary to conduct clinical trials to overcome the potential limitation of TACE with small-caliber DEBs $(<100 \mu \mathrm{m})$. Prospective multicenter studies with a larger number of HCC patients, different bead sizes, and 
Table 2 Evaluation of Tumor Responses on CT and MR Imaging of HCC Treated with DEB-TACE and cTACE Based on mRECIST Criteria

\begin{tabular}{|c|c|c|}
\hline & DEB-TACE & CTACE \\
\hline \multicolumn{3}{|l|}{ mRECIST on CT after TACE } \\
\hline Complete response (CR) & $\begin{array}{l}\text { Entire cystic low-density of the tumor, no arterial- } \\
\text { enhancing part }\end{array}$ & $\begin{array}{l}\text { Homogeneous high-density of lipiodol retention, no } \\
\text { arterial-enhancing part. }\end{array}$ \\
\hline Partial response (PR) & $\begin{array}{l}\text { (I) }>70 \% \text { of tumor features cystic low-density } \\
\text { (2) Residual peripheral arterial-enhancing part, with } \\
\text { early wash-out }\end{array}$ & $\begin{array}{l}\text { Inhomogeneous high-density of retained lipiodol with } \\
\text { residual arterial-enhancing part. } \\
\text { Lipiodol defect may indicate residual tumor despite } \\
\text { absence of arterial enhancement or early wash-out } \\
\text { Dense retained lipiodol may affect early identification of } \\
\text { viable tumor }\end{array}$ \\
\hline \multicolumn{3}{|l|}{ mRECIST on MR after TACE } \\
\hline \multirow[t]{3}{*}{ Complete response (CR) } & $\begin{array}{l}\text { TI-weighted image: } \\
\text { Homogeneous low signal intensity of entire tumor }\end{array}$ & $\begin{array}{l}\text { TI-weighted images: } \\
\text { Homogeneous low signal intensity of entire tumor }\end{array}$ \\
\hline & $\begin{array}{l}\text { T2-weighted image: } \\
\text { High signal intensity of entire tumor suggesting total } \\
\text { necrosis }\end{array}$ & $\begin{array}{l}\text { T2-weighted image: } \\
\text { (1) Homogeneously high signal intensity of entire tumor; } \\
\text { or } \\
\text { (2) Heterogeneous signal intensity due to tumor necrosis }\end{array}$ \\
\hline & $\begin{array}{l}\text { Dynamic study: } \\
\text { No arterial-enhancement or early wash-out }\end{array}$ & $\begin{array}{l}\text { Dynamic study: } \\
\text { No arterial-enhancement or early wash-out }\end{array}$ \\
\hline \multirow[t]{3}{*}{ Partial response (PR) } & $\begin{array}{l}\text { TI-weighted image: } \\
\text { Low signal intensity of entire tumor, and viable } \\
\text { tumors are indistinguishable in this sequence }\end{array}$ & $\begin{array}{l}\text { TI-weighted image: } \\
\text { Low signal intensity of whole tumor, and viable tumors are } \\
\text { indistinguishable in this sequence }\end{array}$ \\
\hline & $\begin{array}{l}\text { T2-weighted image: } \\
\text { Viable tumor may feature hypointense to necrotic } \\
\text { part }\end{array}$ & $\begin{array}{l}\text { T2-weighted image: } \\
\text { Viable tumor may feature hypointense to necrotic part }\end{array}$ \\
\hline & $\begin{array}{l}\text { Dynamic study: } \\
70 \% \text { of tumor features low signal intensity on } \\
\text { precontrast images } \\
\text { Residual tumor features enhancement in arterial } \\
\text { phase with early wash-out }\end{array}$ & $\begin{array}{l}\text { Dynamic study: } \\
70 \% \text { of tumor features low signal intensity on precontrast } \\
\text { images } \\
\text { Residual tumor features enhancement in arterial phase } \\
\text { with early wash-out }\end{array}$ \\
\hline
\end{tabular}

Abbreviations: DEB-TACE, drug-eluting beads transarterial chemoembolization; cTACE, conventional lipiodol-based transarterial chemoembolization; mRECIST, modified response evaluation criteria in solid tumor.

longer follow-up periods are required to validate the clinical efficacy of DEB-TACE treatment for HCC.

Acknowledgements

This study was supported by a research grant from the Tri-Service General Hospital (TSGH-C108-055) and the Ministry of Sciences and Technology (MOST 109-2314B-016-012), Taiwan. The authors want to appreciate the Cancer Registry Group of Tri-Service General Hospital for the clinical data support.

\section{Disclosure}

All the authors report no conflicts of interest in this work.

\section{References}

1. Lencioni R. Chemoembolization in patients with hepatocellular carcinoma. Liver Cancer. 2012;1(1):41-50. doi:10.1159/000339019

2. Prajapati HJ, Xing M, Spivey JR, et al. Survival, efficacy, and safety of small versus large doxorubicin drug-eluting beads TACE chemoembolization in patients with unresectable HCC. AJR Am J Roentgenol. 2014;203(6):W706-W714. doi:10.2214/ajr.13.12308

3. Padia SA, Shivaram G, Bastawrous S, et al. Safety and efficacy of drug-eluting bead chemoembolization for hepatocellular carcinoma: comparison of small-versus medium-size particles. $J$ Vasc Interv Radiol. 2013;24(3):301-306. doi:10.1016/j.jvir.2012.11.023

4. Lin CY, Liu YS, Pan KT, Chen CB, Hung CF, Chou CT. The short-term safety and efficacy of TANDEM microspheres of various sizes and doxorubicin loading concentrations for hepatocellular carcinoma treatment. Sci Rep. 2021;11(1):12277. doi:10.1038/s41598-021-91021-9 
5. Odisio BC, Ashton A, Yan Y, et al. Transarterial hepatic chemoembolization with 70-150 $\mu \mathrm{m}$ drug-eluting beads: assessment of clinical safety and liver toxicity profile. J Vasc Interv Radiol. 2015;26 (7):965-971. doi:10.1016/j.jvir.2015.03.020

6. Zhang L, Sun JH, Ji JS, et al. Imaging changes and clinical complications after drug-eluting beads versus conventional transarterial chemoembolization for unresectable hepatocellular carcinoma: multicenter study. AJR Am J Roentgenol. 2020. doi:10.2214/ajr.20.24708

7. Lee KH, Liapi E, Vossen JA, et al. Distribution of iron oxide-containing embosphere particles after $t$ ranscatheter arterial embolization in an animal model of liver cancer: evaluation with MR imaging and implication for therapy. $J$ Vasc Interv Radiol. 2008;19(10):1490-1496. doi:10.1016/j.jvir.2008.06.008

8. Yamamoto A, Imai S, Kobatake M, et al. Evaluation of tris-acryl gelatin microsphere embolization with monochromatic $\mathrm{X}$ rays: comparison with polyvinyl alcohol particles. J Vasc Interv Radiol. 2006;17(11 Pt 1):1797-1802. doi:10.1097/01.RVI.0000243614.87529.b0

9. Nouri YM, Kim JH, Yoon HK, et al. Update on transarterial chemoembolization with drug-eluting microspheres for hepatocellular carcinoma. Korean J Radiol. 2019;20(1):34 49. doi:10.3348/kjr.2018.0088

10. de Baere T, Plotkin S, Yu R, et al. An in vitro evaluation of four types of drug-eluting microspheres loaded with doxorubicin. J Vasc Interv Radiol. 2016;27(9):1425-1431. doi:10.1016/j.jvir.2016.05.015

11. Lencioni R, de Baere T, Burrel M, et al. Transcatheter treatment of hepatocellular carcinoma with Doxorubicin-loaded DC Bead (DEBDOX): technical recommendations. Cardiovasc Intervent Radiol. 2012;35(5):980-985. doi:10.1007/s00270-011-0287-7

12. Lencioni R, Petruzzi P, Crocetti L. Chemoembolization of hepatocellular carcinoma. Semin Intervent Radiol. 2013;30(1):3-11. doi:10.1055/s-0033-1333648

13. Lewis AL, Gonzalez MV, Lloyd AW, et al. DC bead: in vitro characterization of a drug-delivery device for transarterial chemoembolization. J Vasc Interv Radiol. 2006;17(2 Pt 1):335-342. doi:10.1097/01.Rvi.0000195323.46152.B3

14. Wei G, Yang J. Advances in the treatment of hepatocellular carcinoma using drug-eluting beads. J Interv Med. 2020;3(3):122-127. doi:10.1016/j.jimed.2020.07.003

15. Emerich DF, Snodgrass P, Lafreniere D, et al. Sustained release chemotherapeutic microspheres provide superior efficacy over systemic therapy and local bolus infusions. Pharm Res. 2002;19 (7):1052-1060. doi:10.1023/a:1016434926649

16. Namur J, Citron SJ, Sellers MT, et al. Embolization of hepatocellular carcinoma with drug-eluting beads: doxorubicin tissue concentration and distribution in patient liver explants. $J$ Hepatol. 2011;55 (6):1332-1338. doi:10.1016/j.jhep.2011.03.024

17. Lammer J, Malagari K, Vogl T, et al. Prospective randomized study of doxorubicin-eluting-bead embolization in the treatment of hepatocellular carcinoma: results of the PRECISION V study. Cardiovasc Intervent Radiol. 2010;33(1):41-52. doi:10.1007/s00270-009-9711-7

18. Monier A, Guiu B, Duran R, et al. Liver and biliary damages following transarterial chemoembolization of hepatocellular carcinoma: comparison between drug-eluting beads and lipiodol emulsion. Eur Radiol. 2017;27(4):1431-1439. doi:10.1007/s00330016-4488-y

19. Dinca H, Pelage J, Baylatry $M$, et al. Why do small size doxorubicin-eluting microspheres induce more tissue necrosis than larger ones? A comparative study in healthy pig liver (oral communication 2206-2). 2012.

20. Malagari K, Pomoni M, Moschouris H, et al. Chemoembolization of hepatocellular carcinoma with HepaSphere 30-60 $\mu \mathrm{m}$. Safety and efficacy study. Cardiovasc Intervent Radiol. 2014;37(1):165-175. doi:10.1007/s00270-013-0777-x

21. de Baere T, Guiu B, Ronot M, et al. Real life prospective evaluation of new drug-eluting platform for chemoembolization of patients with Hepatocellular Carcinoma: PARIS registry. Cancers. 2020;12(11): Nov. doi:10.3390/cancers12113405
22. Deipolyi AR, Oklu R, Al-Ansari S, et al. Safety and efficacy of 70$150 \mu \mathrm{m}$ and $100-300 \mu \mathrm{m}$ drug-eluting bead transarterial chemoembolization for hepatocellular carcinoma. $J$ Vasc Interv Radiol. 2015;26(4):516-522. doi:10.1016/j.jvir.2014.12.020

23. Huo YR, Xiang H, Chan MV, et al. Survival, tumour response and safety of $70-150 \mu \mathrm{m}$ versus $100-300 \mu \mathrm{m}$ doxorubicin drug-eluting beads in transarterial chemoembolisation for hepatocellular carcinoma. $J$ Med Imaging Radiat Oncol. 2019;63(6):802-811. doi:10.1111/1754-9485.12971

24. Guiu B, Colombat S, Piron L, et al. Transarterial chemoembolization of hepatocellular carcinoma with idarubicin-loaded tandem drug-eluting embolics. Cancers. 2019;11(7):987. doi:10.3390/cancers11070987

25. Lencioni R, Jm L. Modified RECIST (mRECIST) assessment for hepatocellular carcinoma. Semin Liver Dis. 2010;30(1):52-60. doi:10.1055/s-0030-1247132

26. Vincenzi B, Di Maio M, Silletta M, et al. Prognostic relevance of objective response according to EASL criteria and mRECIST criteria in hepatocellular carcinoma patients treated with loco-regional therapies: a literature-based meta-analysis. PLoS One. 2015;10(7): e0133488. doi:10.1371/journal.pone.0133488

27. Kim BK, Kim SU, Kim KA, et al. Complete response at first chemoembolization is still the most robust predictor for favorable outcome in hepatocellular carcinoma. J Hepatol. 2015;62(6):1304-1310. doi:10.1016/j.jhep.2015.01.022

28. Hwang S, Lee SG, Lee YJ, et al. Prognostic impact of sarcomatous change of hepatocellular carcinoma in patients undergoing liver resection and liver transplantation. $J$ Gastrointest Surg. 2008;12 (4):718-724. doi:10.1007/s11605-007-0393-7

29. Kojiro M, Sugihara S, Kakizoe S, et al. Hepatocellular carcinoma with sarcomatous change: a special reference to the relationship with anticancer therapy. Cancer Chemother Pharmacol. 1989;23(S1):S4S8. doi:10.1007/bf00647229

30. Zen C, Zen Y, Mitry RR, et al. Mixed phenotype hepatocellular carcinoma after transarterial chemoembolization and liver transplantation. Liver Transplant. 2011;17(8):943-954. doi:10.1002/1t.22314

31. Miyayama S, Matsui O, Zen Y, et al. Portal blood supply to locally progressed hepatocellular carcinoma after transcatheter arterial chemoembolization: observation on CT during arterial portography. Hepatol Res. 2011;41(9):853-866. doi:10.1111/j.1872-034X.2011.00836.x

32. Raoul JL, Park JW, Kang YK, et al. Using modified RECIST and alpha-fetoprotein levels to assess treatment benefit in hepatocellular carcinoma. Liver Cancer. 2014;3(3-4):439-450. doi:10.1159/000343872

33. Bruix J, Reig M, Rimola J, et al. Clinical decision making and research in hepatocellular carcinoma: pivotal role of imaging techniques. Hepatology. 2011;54(6):2238-2244. doi:10.1002/hep.24670

34. Golfieri R, Cappelli A, Cucchetti A, et al. Efficacy of selective transarterial chemoembolization in inducing tumor necrosis in small $(<5 \quad \mathrm{~cm})$ hepatocellular carcinomas. Hepatology. 2011;53 (5):1580-1589. doi:10.1002/hep. 24246

35. Miyayama S. Ultraselective conventional transarterial chemoembolization: when and how? Clin Mol Hepatol. 2019;25(4):344-353. doi:10.3350/cmh.2019.0016

36. Manini MA, Sangiovanni A, Martinetti L, et al. Transarterial chemoembolization with drug-eluting beads is effective for the maintenance of the Milan-in status in patients with a small hepatocellular carcinoma. Liver Transplant. 2015;21(10):1259-1269. doi:10.1002/lt.24196

37. Hiraoka A, Kumada T, Kudo M, et al. Hepatic function during repeated TACE procedures and prognosis after introducing sorafenib in patients with unresectable hepatocellular carcinoma: multicenter analysis. Dig Dis. 2017;35(6):602-610. doi:10.1159/000480256

38. Sieghart W, Hucke F, Peck-Radosavljevic M. Transarterial chemoembolization: modalities, indication, and patient selection. $J$ Hepatol. 2015;62(5):1187-1195. doi:10.1016/j.jhep.2015.02.010

39. Moustafa AS, Abdel Aal AK, Ertel N, et al. Chemoem J. Hepatol ply: anatomic and technical considerations. Radiographics. 2017;37 (3):963-977. doi:10.1148/rg.2017160122 
40. Watanabe $\mathrm{Y}$, Tokue $\mathrm{H}$, Taketomi-Takahashi A, et al. Imaging findings and complications of transcatheter interventional treatments via the inferior phrenic arteries in patients with hepatocellular carcinoma. Eur J Radiol Open. 2018;5:171-176. doi:10.1016/ j.ejro.2018.08.010

41. Chiu SH, Chang PY, Shih YL, et al. Efficacy and safety of supplemental transarterial chemoembolization through extrahepatic collateral arteries with drug-eluting beads: treatment for unresectable hepatocellular carcinoma. Drug Des Devel Ther. 2020;14:5029-5041. doi:10.2147/dddt.S266470

42. Omata M, Cheng AL, Kokudo N, et al. Asia-Pacific clinical practice guidelines on the management of hepatocellular carcinoma: a 2017 update. Hepatol Int. 2017;11(4):317-370. doi:10.1007/s12072-0179799-9

43. Bercu ZL, Kim E, Fischman AM, et al. Abstract No. 258 - Decreased efficacy of transarterial chemoembolization in atypical hepatocellular carcinoma. J Vasc Interv Radiol. 2013;24(4,Supplement):S114. doi:10.1016/j.jvir.2013.01.282

44. Shi Q, Chen D, Zhou C, et al. Drug-Eluting beads versus lipiodol transarterial chemoembolization for the treatment of hypovascular hepatocellular carcinoma: a single-center retrospective study. Cancer Manag Res. 2020;12:5461-5468. doi:10.2147/cmar. $\mathrm{S} 255960$

45. Bruix J, Sherman M, Llovet JM, et al. Clinical management of hepatocellular carcinoma. Conclusions of the Barcelona-2000 EASL conference. European Association for the study of the liver. $J$ Hepatol. 2001;35(3):421-430. doi:10.1016/s01688278(01)00130-1
46. Liu L, Wang W, Chen $\mathrm{H}$, et al. EASL- and mRECIST-evaluated responses to combination therapy of sorafenib with transarterial chemoembolization predict survival in patients with hepatocellular carcinoma. Clin Cancer Res. 2014;20(6):1623-1631. doi:10.1158/ 1078-0432.Ccr-13-1716

47. Gillmore R, Stuart S, Kirkwood A, et al. EASL and mRECIST responses are independent prognostic factors for survival in hepatocellular cancer patients treated with transarterial embolization. J Hepatol. 2011;55(6):1309-1316. doi:10.1016/j.jhep.2011.03.007

48. Kloeckner R, Otto G, Biesterfeld S, et al. MDCT versus MRI assessment of tumor response after transarterial chemoembolization for the treatment of hepatocellular carcinoma. Cardiovasc Intervent Radiol. 2010;33(3):532-540. doi:10.1007/s00270-009-9728-y

49. Ozkavukcu E, Haliloğlu N, Erden A. Post-treatment MRI findings of hepatocellular carcinoma. Diagn Interv Radiol. 2009;15(2):111-120.

50. Lim HS, Jeong YY, Kang HK, et al. Imaging features of hepatocellular carcinoma after transcatheter arterial chemoembolization and radiofrequency ablation. AJR Am J Roentgenol. 2006;187(4):W3419. doi:10.2214/ajr.04.1932

51. Kamel IR, Bluemke DA, Eng J, et al. The role of functional MR imaging in the assessment of tumor response after chemoembolization in patients with hepatocellular carcinoma. J Vasc Interv Radiol. 2006;17(3):505-512. doi:10.1097/01.Rvi.0000200052.02183.92

52. Bonekamp S, Jolepalem P, Lazo M, et al. Hepatocellular carcinoma: response to TACE assessed with semiautomated volumetric and functional analysis of diffusion-weighted and contrast-enhanced MR imaging data. Radiology. 2011;260(3):752-761. doi:10.1148/radiol.11102330
Journal of Hepatocellular Carcinoma

\section{Publish your work in this journal}

The Journal of Hepatocellular Carcinoma is an international, peerreviewed, open access journal that offers a platform for the dissemination and study of clinical, translational and basic research findings in this rapidly developing field. Development in areas including, but not limited to, epidemiology, vaccination, hepatitis therapy, pathology and molecular tumor classification and prognostication are all considered for publication. The manuscript management system is completely online and includes a very quick and fair peer-review system, which is all easy to use. Visit http://www.dovepress.com/ testimonials.php to read real quotes from published authors. 